\title{
The Helical Superstructure of Intermediate Filaments
}

\author{
Lila Bouzar ${ }^{a}$, Martin Michael Müller ${ }^{b, c}$, René Messina ${ }^{b}$, Bernd \\ Nöding ${ }^{d}$, Sarah Köster ${ }^{d}$, Hervé Mohrbach ${ }^{b, c}$, Igor M. Kulićc \\ a Laboratoire de Physique des Matériaux, USTHB, \\ BP 32 El-Alia Bab-Ezzouar, 16111 Alger, Algeria \\ $b$ Laboratoire de Physique et Chimie Théoriques - UMR 7019, \\ Université de Lorraine, 1 boulevard Arago, 57070 Metz, France \\ c Institut Charles Sadron, CNRS-UdS, 23 rue du Loess, \\ BP 84047, 67034 Strasbourg cedex 2, France and \\ d Institute for X-Ray Physics, University of Goettingen, \\ Friedrich-Hund-Platz 1, 37077 Göttingen, Germany
}

\begin{abstract}
Intermediate filaments are the least explored among the large cytoskeletal elements. We show here that they display conformational anomalies in narrow microfluidic channels. Their unusual behavior can be understood as the consequence of a previously undetected, large scale helically curved superstructure. Confinement in a channel orders the otherwise soft, strongly fluctuating helical filaments and enhances their structural correlations, giving rise to experimentally detectable, strongly oscillating tangent correlation functions. We propose an explanation for the detected intrinsic curving phenomenon - an elastic shape instability that we call autocoiling. The mechanism involves self-induced filament buckling via a surface stress located at the outside of the cross-section. The results agree with ultrastructural findings and rationalize for the commonly observed looped intermediate filament shapes.

PACS numbers: 87.16.aj,82.35.Pq,87.15.-v
\end{abstract}

The integrity and dynamics of biological cells delicately depend on the mechanical response of their cytoskeleteton consisting of actin filaments, microtubules and intermediate filaments (IFs) 1, 2. While they have been experimentally probed in many different ways, a full understanding of their properties still remains a challenge. This is particularly true for the IFs - the least studied of. Experiments probing their elastic response have shown that they are the most flexible and extensible of the filaments of the cytoskeleton [3 5]. Bio-filaments are usually modeled as semiflexible polymers characterized by their persistence length. For vimentin, a prominent member of the IF family, this model was used in combination with various experimental methods to determine values for the persistence length of a few $\mu \mathrm{m}[3,6,7$.

In most of the experiments the IFs interact strongly with a substrate (AFM and EM) and the extracted physical properties depend on the substrate properties the filaments adhere to. A closer look at the experimental micrographs [3, 6, 7] reveals shapes that resemble sinusoidal waves, loops or circular arcs reminiscent of helices confined to a 2D substrate 8,9 . This leads to the suspicion that IFs are not simple enough to be described by a semiflexible chain. To eliminate possible artifacts from adsorption, we have studied individual IFs in quasi twodimensional microfluidic channels where the filaments, despite geometric confinement, are free to rearrange. Averaging over a number of different filaments a persistence length close to $2 \mu \mathrm{m}$ was found previously [10. However, a deeper inspection of the individual filament data, that we will present in this paper, reveals an anomalous behavior which is incongruent with the expected behavior of a semiflexible polymer. For instance, we observe the transient formation of rings and oscillatory shapes (see Fig. 11. However, the most prominent manifestation of this anomaly manifests itself in a strongly oscillating tangent correlation function for individual filaments, in sharp contrast to the behavior characteristic for a semiflexible chain under lateral confinement [11. In this paper we will show that the data can be completely rationalized by assuming that IFs behave like squeezed helical filaments under lateral confinement.

A central observable in our study is the tangent correlation function, $G(s)$, that provides crucial conformational information about the filament's microstructure. More specifically, it is defined as

$$
G(s)=\langle\overline{\cos \phi(s)}\rangle,
$$

where $\phi(s)$ is the tangent angle of the filament at the arc length position $s$, see sketch in Fig. 11(b). $\langle\ldots\rangle$ in Eq. 1 denotes thermal average and (..) the spatial average along the contour length $(L)$, i.e. $f[\phi(s)]=$ $\frac{1}{L-s} \int_{0}^{L-s} d u f[\phi(u+s)-\phi(u)]$, see also Fig. 11(b).

Revisited (unpublished) experimental data on vimentin filaments whose set up is described in detail elsewhere [10] are displayed in Fig. 2. Quasi two-dimensional microfluidic channels of height $h$ and varying lateral confinement $d$ were employed to confine these filaments. Upon limiting the thermal average of $\overline{\cos \phi(s)}$ to an individual filament, striking oscillations in $G(s)$ set in, see Fig. 2. At prescribed height $(h=0.45 \mu \mathrm{m})$, the two profiles in the main body of figure 2 (red and yellow 

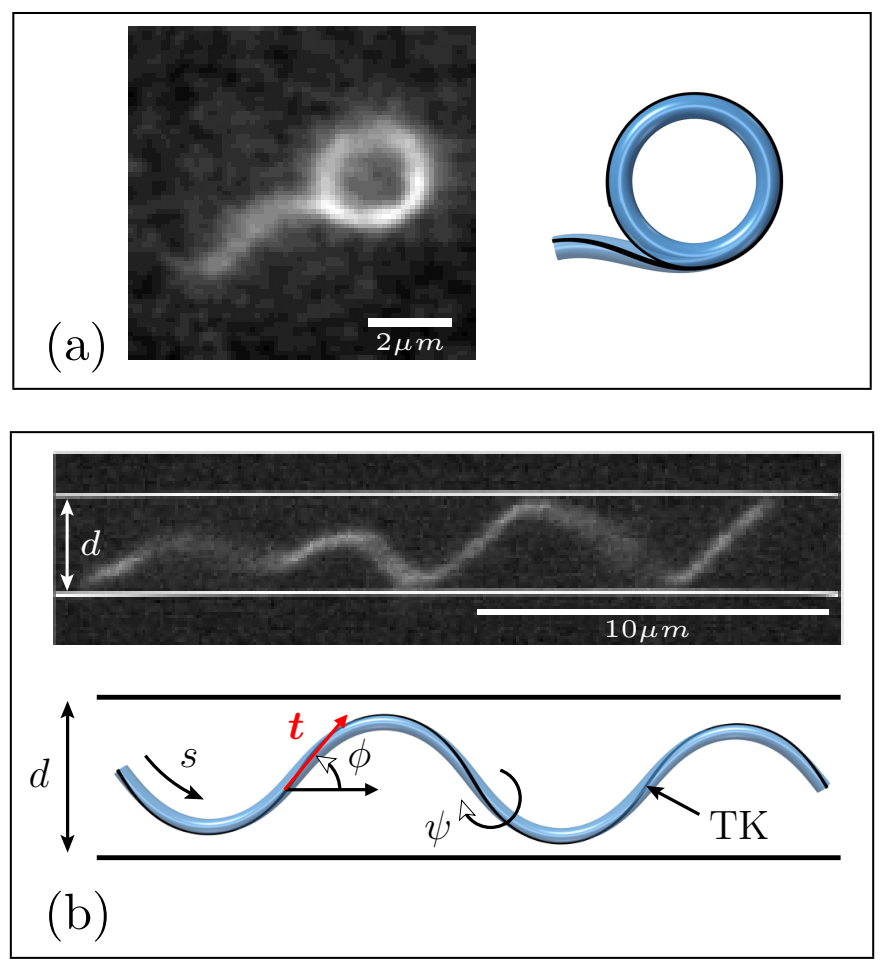

FIG. 1: (a) Left panel: Vimentin filament confined in a slit occasionally coiled up in a transient ring. Right panel: Model three dimensional helical filament getting confined to a plane forming a ring as an energy minimum, see Eq. (2) and text. (b) Upper panel: Typical oscillatory shape of an intermediate filament in a quasi two-dimensional microfluidic channel characterized by a lateral confinement $d[10$. Lower panel: Geometrical setup of the model filament. The tangent vector $\boldsymbol{t}$ evolves with arc-length $s$ along the filament. Its direction is given by the angle $\phi(s)$, measured from the horizontal. $\psi(s)$ denotes the twist angle represented by a black ribbon on the filament's surface. The variation of the twist at curvature inversion points gives rise to conformational defects called twist kinks (TK).

symbols) correspond to two different lateral confinements $(d=1.6 \mu \mathrm{m}$ and $d=2.7 \mu \mathrm{m})$. $G(s)$ increases with decreasing $d$ (i. e., with increasing degree of lateral confinement) as expected, whereas the oscillation amplitudes as well as their associated wave length decrease. These features remain robust upon changing the channel height to $1 \mu \mathrm{m}$, see green circles inset of Fig. 2 It is also possible to observe even stronger and more persistent oscillations, although such conformations are rather rare events, see data with blue circles in inset of Fig. 2 .

In the following, we are going to rationalize these experimental observations by means of Monte Carlo (MC) simulations as well as analytical theory. The underlying idea is that confined IFs can be modeled as a helical superstructure trapped on a flat surface and subject to an additional lateral confinement.

Consider a semi-flexible helical filament of length $L$

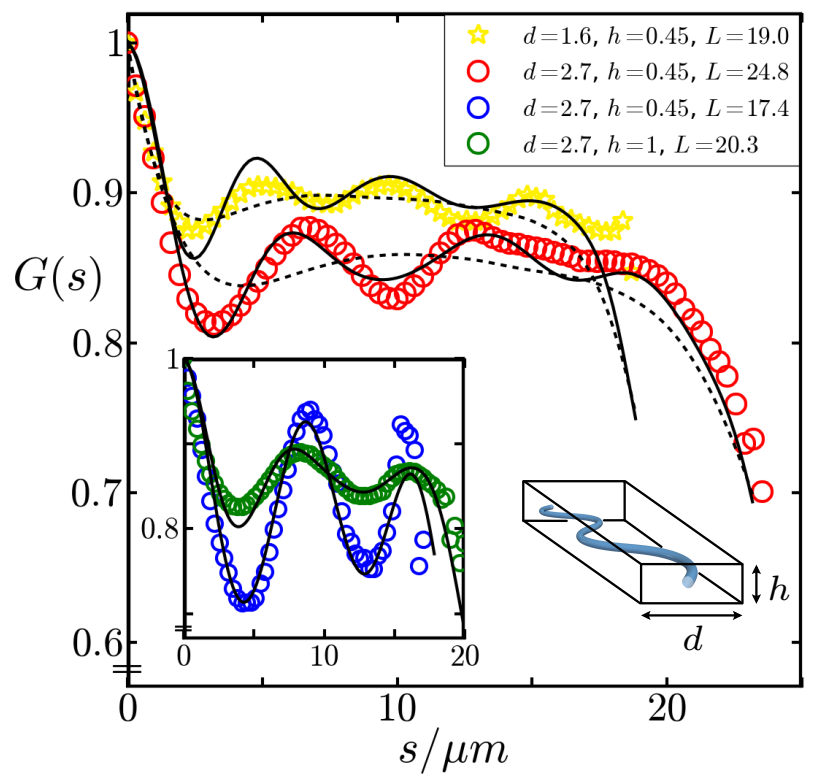

FIG. 2: Tangent correlation functions $G(s)$ of vimentin filaments (experimental data represented by symbols) confined in a quasi two-dimensional microfluidic channel of width $d$ and height $h$ given in microns (as well as $L$ ), see bottom right inset. Bottom left inset: (i) Data for $h=1 \mu \mathrm{m}$ (green circles). (ii) Sample exhibiting strong and persisting oscillations in $G(s)$ (blue circles). Solid (dashed) lines stem from MC simulation data of filaments in two dimensions (i.e., $h=0$ ) with (without) twist.

becoming confined to a plane. Its elastic energy is given by $[8,9$

$$
\begin{aligned}
E= & \frac{1}{2} \int_{0}^{L}\left[B\left(\phi^{\prime}-\omega_{1} \sin \psi\right)^{2}+C\left(\psi^{\prime}-\omega_{3}\right)^{2}\right. \\
& \left.+B \omega_{1}^{2} \cos ^{2} \psi\right] d s,
\end{aligned}
$$

where $\phi^{\prime}(s)=: \kappa(s)$ stands for the curvature, $\psi$ designates the twist angle and $\psi^{\prime}(s)$ the twist with $s$ being the arc-length, see also Fig. 1(b). The constants $B$ and $C$ in Eq. (2) are the bending and torsional stiffness, respectively, $\omega_{1}$ and $\omega_{3}$ are the preferred curvature and twist of the unconfined three-dimensional helical filament [12.

The filament ground state stemming from Eq. (2) obeys two coupled equations: The pendulum-like equation (i) $\psi^{\prime \prime}+\frac{B \omega_{1}^{2}}{2 C} \sin (2 \psi)=0$ and (ii) $\kappa=\omega_{1} \sin \psi$ indicating that curvature is slaved by the twist angle in contrast to the unconfined three-dimensional case (where both decouple). In general, depending on the material parameters, a rich variety of equilibrium shapes resembling loops, waves, spirals or circles exist [9]. As a matter of fact, these shapes can be seen as the result of interacting repulsive conformational defects corresponding to curvature inversion points. In terms of twist, such defects originate from a rapid variation of $\psi(s)$ (reminiscent of a kink) and are called twist-kinks (TKs). A relevant di- 

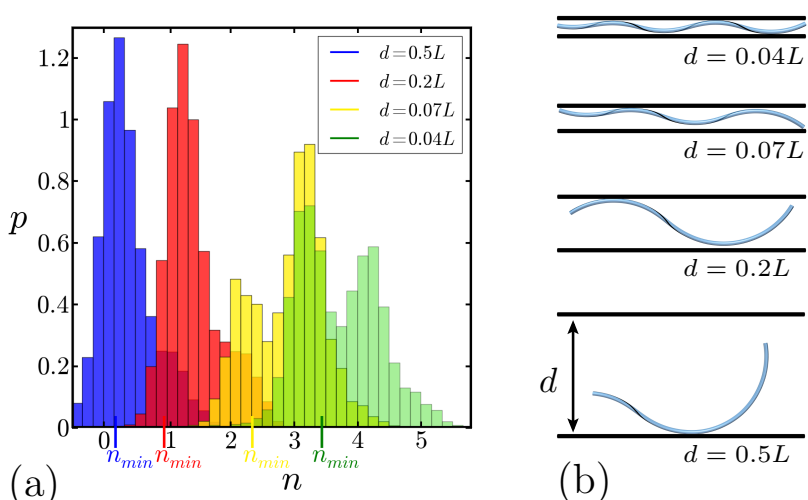

(b)

FIG. 3: (a) Simulation data for the probability density of the number of twist-kinks $n$ for different values of the lateral confinement $d$ with $\omega_{1}=\pi, \gamma=5, B=10, C=0.16$, (with $k_{B} T=L=1$ ). (b) Low tempertaure snapshots of a filament close to its ground state for the same values of $d$ as in the histogram illustrating the twist-kink injection.

mensionless parameter is $\gamma=\frac{4 \omega_{1}^{2} B}{\pi^{2} \omega_{3}^{2} C}$ which measures the ratio of bending and twisting energy. For $\gamma>1$, the ground state approaches a twist-kink-free circular arc of radius $1 / \omega_{1}$, see Fig. 1(a). For $\gamma<1$, the filament can be populated by twist-kinks whose density is limited by their repulsion [25]. Extensive MC simulations based on the Hamiltonian of Eq. (2) have been carried out in order to explore the conformations of confined filaments in full detail. The filament is modelled as a discrete chain made up of $N$ monomers (i.e., segments) of fixed length $b$ [26]. Our best matching MC data for $G(s)$ can be found in Fig. 2. Interestingly, taking into account the twist via Eq. (2) corroborates the experimentally observed oscillations by employing consistent material parameters [27, see Fig. 2 . These results suggest that the unconfined vimentin possesses a helical superstructure characterized by a radius $\sim 0.4 \mu \mathrm{m}$ and pitch $\sim 4 \mu \mathrm{m}$ and a bending persistence length $B /\left(k_{B} T\right) \sim 50 \mu \mathrm{m}[28]$. The microscopic origin of this helix is still unclear but a plausible mechanism is proposed at the end of this paper. In contrast, for twist-free semiflexible chains, no oscillations emerge in $G(s)$ where the usual Odijk behavior [11] is recovered, see dashed lines in Fig. 2.

To deepen our understanding of the role of the internal twist, that is a hidden degree of freedom in the experiments, we further utilize the simulation data to analyze the filament conformation for various degrees of lateral confinement. A useful quantity is the probability density of twist-kinks occurrence, $p(n)$, where $n$ is the number of twist-kinks which is adequately defined as $n=\frac{1}{\pi} \int_{0}^{L} \psi^{\prime}(s) d s$. Probability density profiles $p(n)$ for varying lateral confinement $d$ are depicted in Fig. 3(a). On average, the number of twist-kinks increases with decreasing $d$, see Fig. 3(a). Computing the correspond-

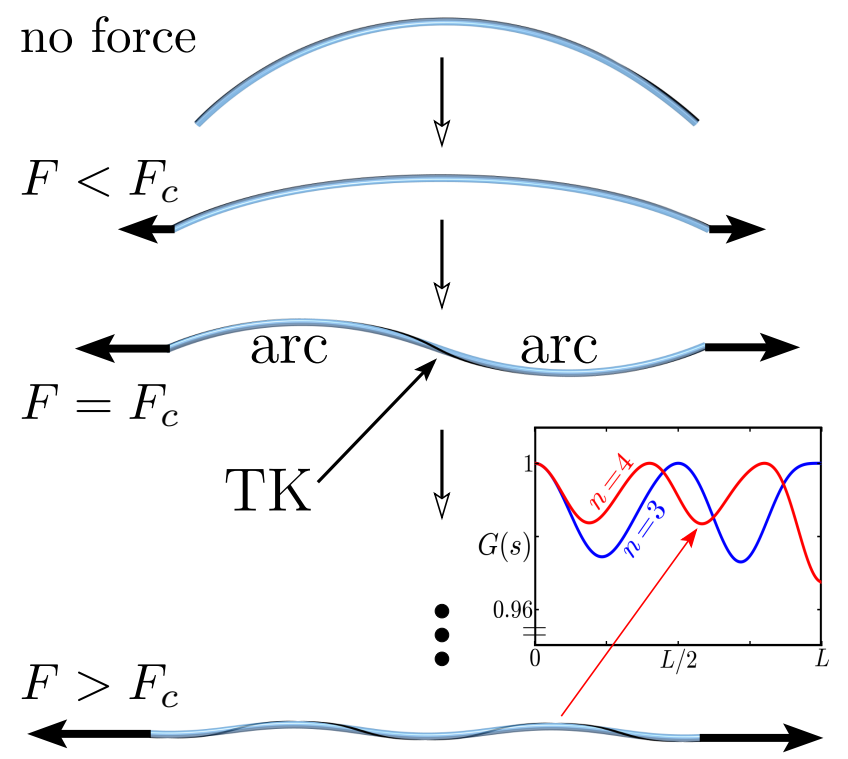

FIG. 4: Evolution of a ground state of an elastic filament under an external pulling force. Inset: Corresponding tangent correlation functions for $n=3$ (blue) and $n=4$ (red) twistkinks (TKs).

ing $G(s)$ (not shown) clearly indicates that the average number of twist-kinks $n$ also describes the number of extrema encountered in $G(s)$. Thermal fluctuations induce injections and ejections of twist-kinks at the ends of the filament, which qualitatively explains the fading of the oscillations in $G(s)$ shown in Fig. 2 Concomitantly, a finite temperature induces a broadening in $p(n)$.

To shed more light on the underlying physical mechanisms of confinement-mediated twist-kink production let us consider a more tractable analytical model. It consists in replacing the confinement by a pulling force at zero temperature, see Fig 4 . Although this systems is different it illustrates the twist-kink injection mechanism in a phenomenological manner. In the presence of the external force $F$ and for small deformations $(\varphi \ll 1)$ the elastic energy becomes

$$
E_{F} \simeq E+\frac{F}{2} \int_{0}^{L} \phi^{2}(s) d s
$$

Consider the regime $\gamma \gg 1$ which has as a ground state (in absence of a force) a circular arc $\phi(s)=-\omega_{1} s$ and energy $E_{0}=C L \omega_{3}^{2} / 2$, see Fig. 4 The formation of a twist-kink is energetically favorable when the pulling force exceeds a critical value $F_{c}=\frac{64}{L^{3} \omega_{1}} \sqrt{B C}$. The latter is the result of comparison of the elastic energy of a twistkink-free filament $\left(E_{0}+\frac{\omega_{1}^{2} L^{3}}{24} F\right)$ with that of a filament containing a single twist-kink located at the midpoint $\left(E_{0}+\omega_{1} \sqrt{B C}+\frac{\omega_{1}^{2} L^{3}}{96} F\right)$, see Fig. 4. In the opposite, small force regime $\left(F<F_{c}\right)$, the filament deforms like a stretched elastic arc, see Fig. 4. 
Above $F_{c}$, one or more twist-kinks are formed, see Fig. 4. The required force to nucleate $n$ twist-kinks is given by $F_{c}(n)=\frac{48 \sqrt{B C}}{L^{3} \omega_{1}} \frac{n^{2}(n+1)^{2}}{2 n+1}$. Note, that the kinks mutually repel each other giving rise to an ordered one-dimensional crystal-like structure of $n$ twist-kinks separated by circular arcs of switching curvature, see Fig. 4. Expanding the chain conformation in Fourier modes, and keeping only the dominant one we extract the tangent correlation function:

$$
G(s) \approx 1-\frac{16 \ell^{2} \omega_{1}^{2}}{\pi^{4}} \sin ^{2}\left(\frac{\pi s}{2 \ell}\right)\left(1+\frac{\ell \sin (\pi s / \ell)}{\pi(L-s)}\right)
$$

with $\ell=L /(n+1)$ representing the distance between two adjacent twist-kinks. Typical profiles of $G(s)$, displaying oscillations similar to the confinement case, are shown in Fig. 4. Simulation results with external force, not shown here, corroborate these findings.

Thus experimental and theoretical results strongly suggest that vimentin filaments have a helical superstructure, indicated by remarkable oscillations in the tangent correlation function in confined geometry.

The molecular origin of the helical shape of vimentin filaments is unclear at this point. Oscillations in the tangent correlation functions of individual actin filaments were also previously observed [17, 18, and helical superstructures of microtubule reported [19, 20]. Thus, the question arises which general physical mechanism could be at the origin of the curved/helical shapes of those biofilaments. Here we propose a mechanism based on self-buckling, for which we suggest the name autocoiling, see Fig. 5. It is a generic feature of filaments exposed to surface stresses that induce a broken symmetry and thus curved states. It can be witnessed in the mundane example of a drying spaghetti, see Fig. 5(c). The outer layer of the spaghetti dries and shrinks faster and induces a buckling stress on the (transiently) more swollen core.

For vimentim, there are a few hints towards a surface stress from its molecular structure. During the assembly of its constituent monomers the monomers form highly elongated coiled-coil dimers, then tetramers and at an intermediate stage give rise to an approximately "spindle shaped" 32-mer (see images in Ref. 21) - the so called unit length filament (ULF) 22. These ULFs then assemble into long filaments. The initially rugged, "rough" filaments of spindle-like ULF subunits, longitudinally anneal and the filaments undergo a maturation phase during which the surface smoothens Ref. [21, 22]. We suggest that the spindle shape and the finite lateral thickness of the ULF can be understood as originating from double-twist-frustration caused by chiral (cholesteric) interactions of the coiled-coil alpha helices aligned along the axis. As proposed by Grason and Bruinsma [23, a bundle of chiral objects displays a finite size as the chains on the outside are progressively more tilted than those on the inside. This tilt causes a surface stress and axial shortening of the outer chains with respect to the inner

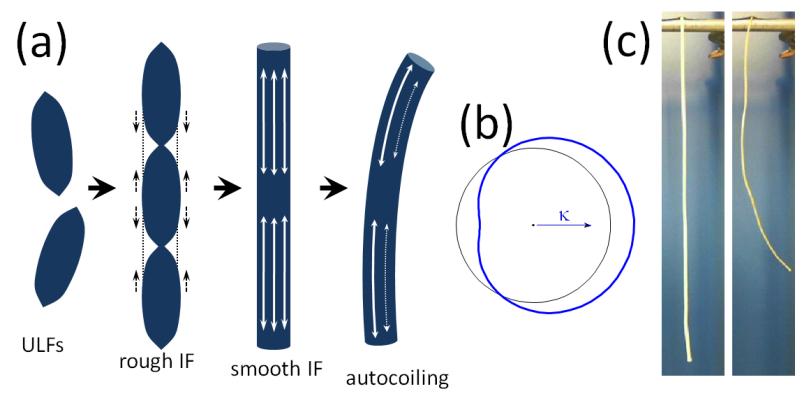

FIG. 5: a) Stages of assembly of IFs and their autocoiling instability. b) The cross-section deformation of the bent circular rod. c) The autocoiling of drying spaghetti.

ones. As the simplest possible model for self-buckling under a surface stress we consider an isotropic incompressible elastic rod of radius $R$ and length $L$. Its energy is $U=\frac{1}{2} \int d V \sigma_{i j} \varepsilon_{i j}+\lambda \Delta S$ where $\sigma_{i j}$ and $\varepsilon_{i j}$ are the bulk stress and strain tensor respectively. The constant $\lambda>0$ is an isotropic tensile surface stress (surface energy density). $\Delta S$ denotes the variation of the surface after deformation. When negative, the term $\lambda \Delta S$ can compete with the positive elastic bulk energy.

Under a pure bending deformation with a constant curvature $\kappa$ of the rod, the cross-section is slightly deformed (see $5(\mathrm{a})$ ) and $\Delta S<0$ as deduced from the deformation field of a bent rod [24]. It can be shown that $\frac{\Delta S}{S_{0}} \approx$ $-\frac{3}{16} R^{2} \kappa^{2}$ where $S_{0}=2 \pi L R$ is the surface of the undeformed rod. The total surface+elastic energy can then be written as $U=\frac{1}{2} B_{e}(\lambda) L \kappa^{2}$ with an effective bending modulus that has contributions from both the bulk and the surface stress of the $\operatorname{rod}: B_{e}(\lambda)=\frac{\pi}{4} Y R^{4}-\lambda \frac{3 \pi}{4} R^{3}$ where $Y$ is the Young modulus. For sufficently large $\lambda$ the stiffness $B_{e}(\lambda)$ vanishes and the rod becomes unstable. This is the signature of a spontaneous broken symmetry of the rod, i.e., self-buckling.

Beyond the curvature, the origin of helical torsion of vimentin, its magnitude and handedness deserve further theoretical and experimental investigations. At this point we speculate that the handedness and the pitch of IFs are inherited from the chiral inter-alpha-helix interaction on the monomer level.

In summary, we report experimental and theoretical evidence for a helical superstructure in intermediate filaments. Lateral confinement in a quasi-2D channel orders the otherwise soft and strongly fluctuating twist-kink defects and leads to experimentally detectable, oscillating tangent correlation functions. The underlying physical mechanism is the injection of low energy defects, that can be seen as a new deformation mode for confined helical filaments. This behavior is reminiscent of the plastic deformations mediated by dislocations in solids [16]. The self-buckling mechanism suggested to be at the origin of the helical states of vimentin could also be common in other biological filaments beyond IFs. More generally, 
tensile surface stress could be induced by any mismatch between the surface and the bulk of the filament, like e.g. the surface tension between the ordered water and ions at the outer layers of the filament that display mismatched osmotic and Maxwell stresses between the interior and exterior.

The biological meaning of an intrinsically stiffer but coiled helical structure is apparent. A coiled structure resists small forces by uncoiling and displays high compliance without damage up to the point of stronger elongation. This reinforces the IFs' natural role as supporting mechanical elements and protective stress absorbers of the cell.

[1] B. Alberts, et al. Molecular Biology of the Cell . 4th edition. New York: Garland Science; 2002.

[2] A. Amos and W. G. Amos, Molecules of the Cytoskeleton, Guilford, New York, 1991.

[3] N. Mücke et al., Assessing the Flexibility of Intermediate Filaments by Atomic Force Microscopy, J. Mol. Biol. 335, 1241 (2004).

[4] L. Kreplak, H. Bär, J. F. Leterrier, H. Hermann, U. Aebi, Exploring the Mechanical Behavior of Single Intermediate Filaments, J. Mol. Biol. 354, 569 (2005).

[5] L. Kreplak, H. Hermann, U. Aebi, Tensile Properties of Single Desmin Intermediate Filaments, Biophys. J. 94, 2790 (2008).

[6] S. Winheim et al., Deconstructing the Late Phase of Vimentin Assembly by Total Internal Reflection Fluorescence Microscopy (TIRFM), PLoS ONE 6, e19202 (2011).

[7] M. Schopferer et al., Desmin and Vimentin Intermediate Filament Networks: Their Viscoelastic Properties Investigated by Mechanical Rheometry, J. Mol. Biol. 388, 133 (2009).

[8] G.-M. Nam, N.-K. Lee, H. Mohrbach, A. Johner, I. M. Kulić, Helices at Interfaces, Europhys. Lett. 100, 28001 (2012).

[9] L. Bouzar, M. M. Müller, P. Gosselin, I. M. Kulić, H. M. Mohrbach, Squeezed helical elastica, Eur. Phys. J. E 39, 114 (2016).

[10] B. Nöding and Sarah Köster, Intermediate Filaments in Small Configuration Spaces, Phys. Rev. Lett. 108, 088101 (2012).

[11] T. Odijk, The statistics and dynamics of confined or entangled stiff polymers, Macromolecules, 16, 1340 (1983)

[12] N. Chouaieb, A. Goriely, and J. H. Maddocks, Helices, Proc. Natl. Acad. Sci. U.S.A. 103, 9398 (2006).

[13] N. Madras and A. D. Sokal, The pivot algorithm: A highly efficient Monte Carlo method for the self-avoiding walk, J. Stat. Phys. 50, 109 (1988).

[14] M. P. Allen and D. J. Tildesley, Computer Simulations of Liquids, Clarendon Press (1987).

[15] J. Block et al., Nonlinear Loading-Rate-Dependent Force Response of Individual Vimentin Intermediate Filaments to Applied Strain, Phys. Rev. Lett. 118, 048101 (2017).

[16] J. Friedel, R. Smoluchowski and N. Kurti, Dislocations, International Series of Monographs on Solid State Physics, 1964.

[17] S. Köster, J. Kierfeld, and T. Pfohl, Characterization of single semiflexible filaments under geometric constraints, Eur. Phys. J. E 25, 439 (2008).

[18] S. Köster, D. Steinhauser, and T. Pfohl, Brownian motion of actin filaments in confining microchannels, J. Phys.: Cond. Mat. 17, S4091 (2005).

[19] H. Mohrbach, A. Johner, I. M. Kulić, Tubulin Bistability and Polymorphic, Dynamics of Microtubules, Phys. Rev. Lett. 105, 268102 (2010).

[20] H. Mohrbach, A. Johner, I. M. Kulić, Cooperative lattice dynamics and anomalous fluctuations of microtubules, Eur. Biophys. J. 41, 217 (2012).

[21] H. Herrmann et al., Characterization of Distinct Early Assembly Units of Different Intermediate Filament Proteins, J. Mol. Biol. 286, 1403 (1999).

[22] H. Herrmann and U. Aebi, Intermediate filaments: Molecular structure, assembly mechanism, and integration into functionally distinct intracellular scaffolds, Annu. Rev. Biochem. 73, 749 (2004).

[23] G.M. Grason and R.F. Bruinsma, Chirality and Equilibrium Biopolymer Bundles, Phys. Rev. Lett. 99, 098101 (2007)

[24] W. M. Lai, D. Rubin, E. Krempl, Introduction to Continuum Mechanics, Elsevier, 2010.

[25] The number of twist-kinks can fluctuate at a finite temperature. The translational zero mode of the injected and moving twist-kinks leads to a chain with anomalously large "hyperflexibility" 8].A remarkable analogy can be established with dislocations in solids, where (plastic) deformation is facilitated by the production of such energetically cheap linear defects in crystals [16].

[26] Each monomer is characterized by four variables $\left(x_{i}, y_{i}, \phi_{i}, \psi_{i}\right)$ with $i=1 \ldots N$. The positions $x_{i}=$ $x_{i-1}+\cos \phi_{i-1}$ and $y_{i}=y_{i-1}+\sin \phi_{i-1}$ stand for the Cartesian coordinates in the plane. One Monte Carlo step consists of $N$ attempted local rotations and torsions of each monomer sequentially and a single attempt of a global translation (only relevant in the presence of the confining channel), rotation [13] and torsion. Standard Metropolis scheme is then used to generate a canonical ensemble [14. Typically, $10^{6}$ Monte Carlo steps were employed for a simulation run and about the second half of the steps was used to gather statistics.

[27] (i) For simulation data with finite twist, the elastic constants are $B /\left(k_{B} T\right) \simeq 50 \mu \mathrm{m}$ and $C /\left(k_{B} T\right) \simeq 3 \mu \mathrm{m} \mathrm{ex}-$ cept for the corresponding experimental data with blue circles where $C /\left(k_{B} T\right) \simeq 10 \mu \mathrm{m}$. The geometrical parameters are typically $\omega_{1}^{-1} \simeq 2 \mu \mathrm{m}$ of $\omega_{3}^{-1} \simeq 1 \mu \mathrm{m}$ (ii) For twist-free filaments (i.e., $\omega_{1}=\omega_{3}=C=0$ ), $B /\left(k_{B} T\right) \simeq 5 \mu \mathrm{m}$.

[28] Note that the value of $B$ is much higher than determined previously from multi-filament averaging - a procedure that neglects single filament correlations and measures an "apparent" persistence length. 